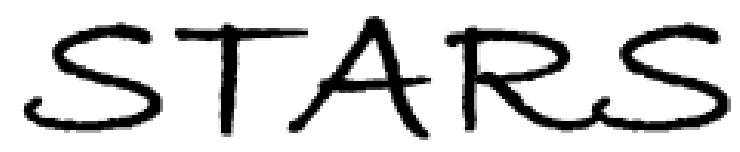

University of Central Florida

STARS

$1-1-1997$

\title{
Intracavity Gain And Absorption Dynamics Of Hybrid Modelocked Semiconductor Lasers Using Multiple Quantum Well Saturable Absorbers
}

\author{
S. Gee \\ University of Central Florida \\ R. Coffie \\ University of Central Florida \\ P. J. Delfyett \\ University of Central Florida \\ G. Alphonse \\ J. Connolly \\ Find similar works at: https://stars.library.ucf.edu/facultybib1990 \\ University of Central Florida Libraries http://library.ucf.edu
}

This Article is brought to you for free and open access by the Faculty Bibliography at STARS. It has been accepted for inclusion in Faculty Bibliography 1990s by an authorized administrator of STARS. For more information, please contactSTARS@ucf.edu.

\section{Recommended Citation}

Gee, S.; Coffie, R.; Delfyett, P. J.; Alphonse, G.; and Connolly, J., "Intracavity Gain And Absorption Dynamics Of Hybrid Modelocked Semiconductor Lasers Using Multiple Quantum Well Saturable Absorbers" (1997). Faculty Bibliography 1990s. 1920.

https://stars.library.ucf.edu/facultybib1990/1920

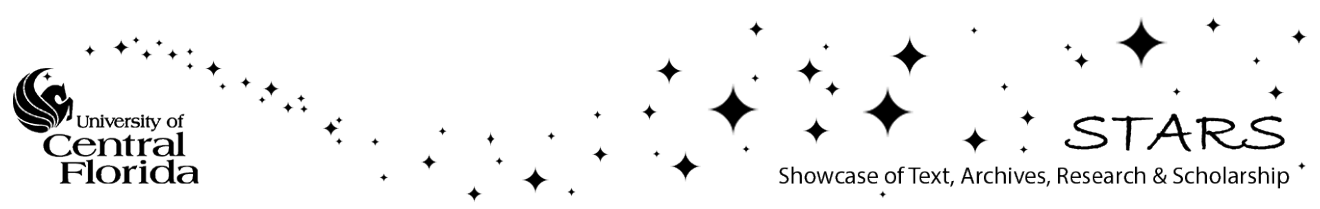




\section{Intracavity gain and absorption dynamics of hybrid modelocked semiconductor lasers using multiple quantum well saturable absorbers}

Cite as: Appl. Phys. Lett. 71, 2569 (1997); https://doi.org/10.1063/1.119333

Submitted: 05 May 1997. Accepted: 03 September 1997. Published Online: 04 June 1998

S. Gee, R. Coffie, P. J. Delfyett, G. Alphonse, and J. Connolly

ARTICLES YOU MAY BE INTERESTED IN

SELF MODE-LOCKING OF LASERS WITH SATURABLE ABSORBERS

Applied Physics Letters 8, 174 (1966); https://doi.org/10.1063/1.1754541

InGaAsP monolithic extended-cavity lasers with integrated saturable absorbers for active, passive, and hybrid mode locking at $8.6 \mathrm{GHz}$

Applied Physics Letters 62, 1445 (1993); https://doi.org/10.1063/1.108653

Ultrafast dynamics in field-enhanced saturable absorbers

Applied Physics Letters 64, 676 (1994); https://doi.org/10.1063/1.111058
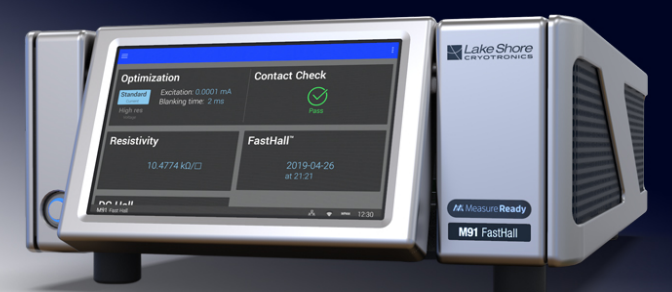

Mn Measure Ready M91 FastHall ${ }^{\text {TM }}$ Controller

A revolutionary new instrument for complete Hall analysis See the video $\bullet$

LakeShore 


\title{
Intracavity gain and absorption dynamics of hybrid modelocked semiconductor lasers using multiple quantum well saturable absorbers
}

\author{
S. Gee, R. Coffie, and P. J. Delfyett ${ }^{\mathrm{a})}$ \\ Department of Electrical \& Computer Engineering and Department of Physics, Center for Research and \\ Education in Optics and Lasers (CREOL), University of Central Florida, Orlando, \\ Florida 32816-2700
}

\author{
G. Alphonse and J. Connolly \\ David Sarnoff Research Center, Princeton, New Jersey 08543-5300
}

(Received 5 May 1997; accepted for publication 3 September 1997)

\begin{abstract}
Time resolved intracavity gain, saturable absorption dynamics, intracavity intensity pulse profiles, and their corresponding spectrograms, were measured in an external cavity hybrid mode-locked semiconductor diode laser. These measurements were performed to obtain fundamental information of the mode-locking dynamics and to determine their role in the pulse shaping and chirping dynamics. The results of these experiments show that the integrating nonlinearity associated with gain depletion, coupled with group velocity dispersion, leads to asymmetric intensity pulse profiles with predominantly cubic temporal phase, while saturable absorption coupled with group velocity dispersion tends to linearize the chirp. Exploitation of these dynamics may allow researchers to generate optical pulses with higher peak intensities than previously reported. (C) 1997 American Institute of Physics. [S0003-6951(97)01244-8]
\end{abstract}

Compact sources of ultrashort, high power optical pulses are necessary for realizing applications in areas of applied nonlinear photonics. Semiconductor lasers are viable candidates owing to their small size, good wall plug efficiency, and robustness. However, the dynamics of semiconductor laser media prevent the production of high power ultrashort optical pulses. ${ }^{1,2}$ In this letter, we present experimental measurements of the intracavity gain and saturable absorber dynamics, to investigate their role in the pulse formation and chirping dynamics in hybrid mode-locked semiconductor diode lasers. To support these measurements, intracavity intensity pulse profiles and their corresponding spectrograms were measured to provide direct experimental observation of the effects suggested by the intracavity dynamics.

The experimental setup is shown in Fig. 1. An external cavity hybrid mode-locked semiconductor laser producing $\sim 700$ fs optical pulses at $300 \mathrm{MHz}$, centered at $830 \mathrm{~nm}$ is the laser system under test. ${ }^{3}$ The output pulses from the laser system are used as the probing pulses and injected into the laser oscillator using a polarizing beam splitter. To monitor the gain and absorption dynamics, the transmitted probe beam is partially deflected from the cavity using a pellicle beam splitter and detected by standard lock-in techniques.

In Fig. 2 is the time resolved intracavity gain measurement. The salient features are the two transient reductions of gain superimposed on a sinusoidally varying gain. The transient gain reductions are owing to the intracavity pulse passing through the semiconductor laser amplifier (SOA). The important observation is that the carrier heating and cooling effects are not observed in the gain dynamics, i.e., the SOA exhibits conventional gain dynamics. This is important because frequency chirping mechanisms such as self-phase modulation are strongly coupled to the gain dynamics. In addition, it should be noted that the gain has recovered to its

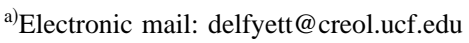

initial value within $350 \mathrm{ps}$, in contrast to the gain recovery time of $1.1 \mathrm{~ns}$. This is owing to the time varying pumping rate associated with the rf bias current. It should also be noted that the pulses do not pass through the SOA device at the point of maximum gain. This is owing to the location of the SOA device with respect to the rear reflector. Since the SOA device is not located at the rear reflector, the displacement of the SOA forces the optical pulses to travel through the SOA at times when the optical gain is approximately equal for each pulse. This manifests itself as the optical pulses passing through the SOA at times symmetrically displaced from the point of maximum gain. In our case, this corresponds to the round trip time between the SOA and the rear reflector.

The time resolved intracavity saturable absorption was also measured. The time resolved reflectance of the multi-

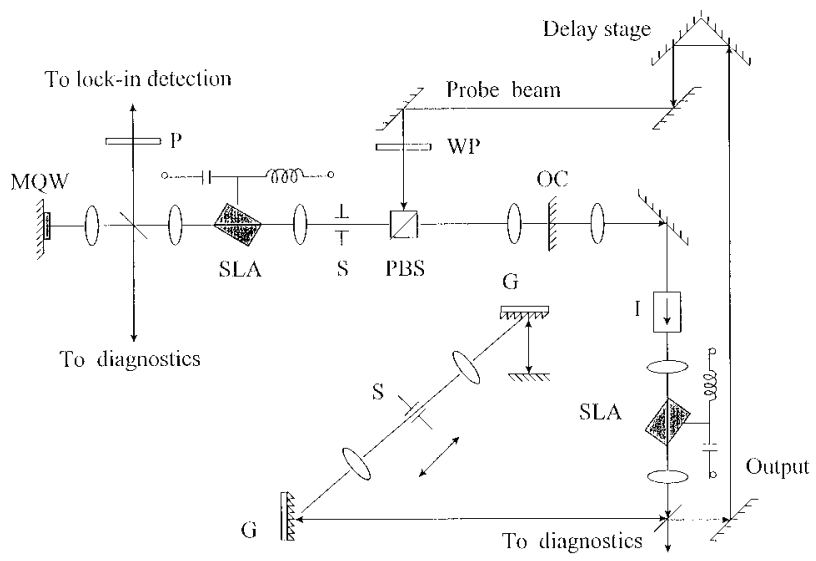

FIG. 1. Experimental setup for intracavity gain dynamic measurement (MQW) multiple quantum well saturable absorber, (PBS) polarizing beam splitter, (SOA) semiconductor laser amplifier, (OC) output coupler, (G) diffraction grating, (S) slit, (I) optical isolator, (P) polarizer, (WP) half wave plate. 


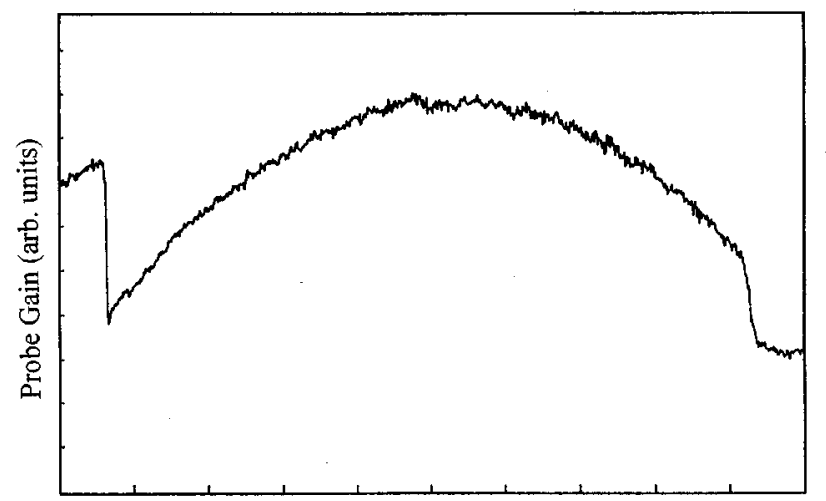

Time Delay (120 psec/div)

FIG. 2. Time resolved intracavity gain dynamic measurement, showing transient gain depletion due to pulse passage through the SOA, and a long term sinusoidal gain recovery owing to the rf modulation frequency

quantum well (MQW) mirror exhibits a relative increase of $20 \%$ with a rise time of $10 \mathrm{ps}$, corresponding to the integrated pulse intensity. The absorber only exhibits a slow recovery; with an exponential absorption recovery time constant of $280 \mathrm{ps.} \mathrm{It} \mathrm{should} \mathrm{be} \mathrm{noted} \mathrm{that} \mathrm{the} \mathrm{recovery} \mathrm{time}$ measured in these experiments differs as compared to previously measured absorber recovery times. ${ }^{4,5}$ These differences may be attributed to differences in the MQW absorber design and differences in the optical transverse mode profile of the lasers used in this versus prior measurements.

To demonstrate the pulse shaping effects induced by the saturable absorber, input and output pulse intensity profiles were measured. This was achieved by cross correlating the intracavity pulses with the compressed output optical pulse. Cross correlation information is then obtained by examining the side lobes of the three-peak correlation signal. In Fig. 3 (top) is the intensity pulse profile of the optical pulse before the saturable absorber, and Fig. 3 (bottom) is the intensity pulse profile of the optical pulse after the saturable absorber. The salient feature is the reduction of the rising edge of the optical pulse from 6 to $3 \mathrm{ps}$; representing a change of nominally $50 \%$ per round trip. It should be noted that the shoul-

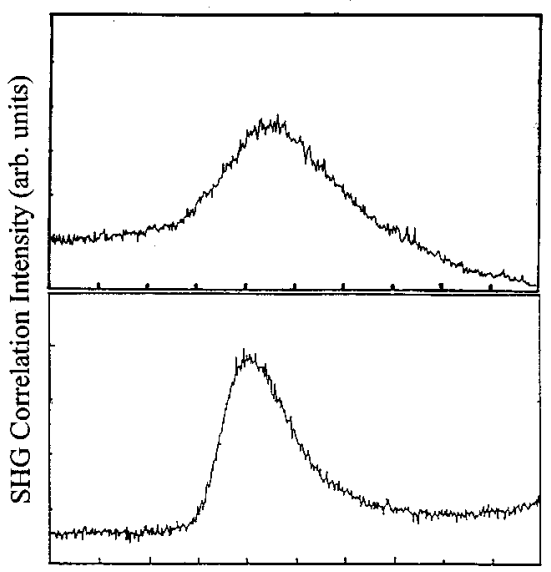

Time Delay (6.4 psec/div)

FIG. 3. Intracavity temporal pulse profiles: (Top) Intensity pulse profile before the intracavity saturable absorber. (Bottom) Intensity pulse profile after the intracavity saturable absorber.

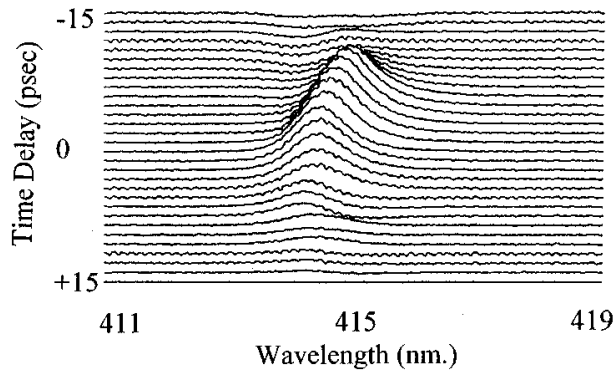

FIG. 4. Spectrally resolved cross correlation of the intracavity pulse measured after the saturable absorber.

ders in the correlation traces are artifacts created by the twopulse correlation technique employed.

In order to assess the effect of the nonlinear dynamics on the chirping properties of the generated optical pulses, the instantaneous frequency of the optical pulses were directly measured by spectrally resolving the cross-correlated intensity profiles at the second harmonic frequency. ${ }^{6,7}$ Those were measured at three locations of the cavity, i.e., the laser output, before the saturable absorber and after the saturable absorber. One of the resulting spectrograms is shown in Fig. 4. In each case, the spectrograms show optical pulses with fast rising edges, with slower trailing edges. These effects were clearly observed in the cross correlation traces above. In addition, the spectrograms show that the instantaneous frequency of the optical pulses are not constant, that is the center or carrier wavelength varies throughout the pulse duration. In these traces, the center wavelength is upchirped and tends to vary linearly over a major portion of the optical pulse. Quantitatively, the spectrograms show a total wavelength variation of $0.8 \mathrm{am}$ at the second harmonic wavelength, implying a total wavelength chirp of $1.6 \mathrm{~nm}$ at the fundamental wavelength. It should be noted that the chirp exists over the duration of the pulse, implying a nonlinear dispersion of $\sim 5 \mathrm{ps} / \mathrm{nm}$.

The intracavity pulse shapes and the corresponding spectrograms can be easily explained once the intracavity nonlinear dynamics are considered. ${ }^{8}$ It has been shown that a pulse propagating through a semiconductor optical amplifier will have a time dependent frequency impressed upon it, where the instantaneous frequency will exactly follow the optical pulse shape ${ }^{8,9}$ This occurs through self-phase modulation, where the nonlinearity is an integrating nonlinearity. Under this condition, the instantaneous frequency varies directly proportional to the optical intensity. The instantaneous frequency, defined as $\omega_{\text {inst }}=\omega-\partial \phi / \partial t$, implies that the predominantly parabolic frequency sweep corresponds to cubic temporal phase. It should be noted that an instantaneous Kerr nonlinearity leads to primarily linear frequency sweep, or quadratic temporal phase. The immediate consequence of the integrating nonlinearity is that, when coupled with group velocity dispersion (GVD), the GVD slows down the blue frequency components at the front and rear of the pulse, while the center portion of the pulse (red) is allowed to propagate towards the front of the pulse, yielding the pulse steepening effect observed. This is in direct contrast to conventional mode-locked lasers with instantaneous nonlinearities, where GVD symmetrically broadens the optical pulse. When the 


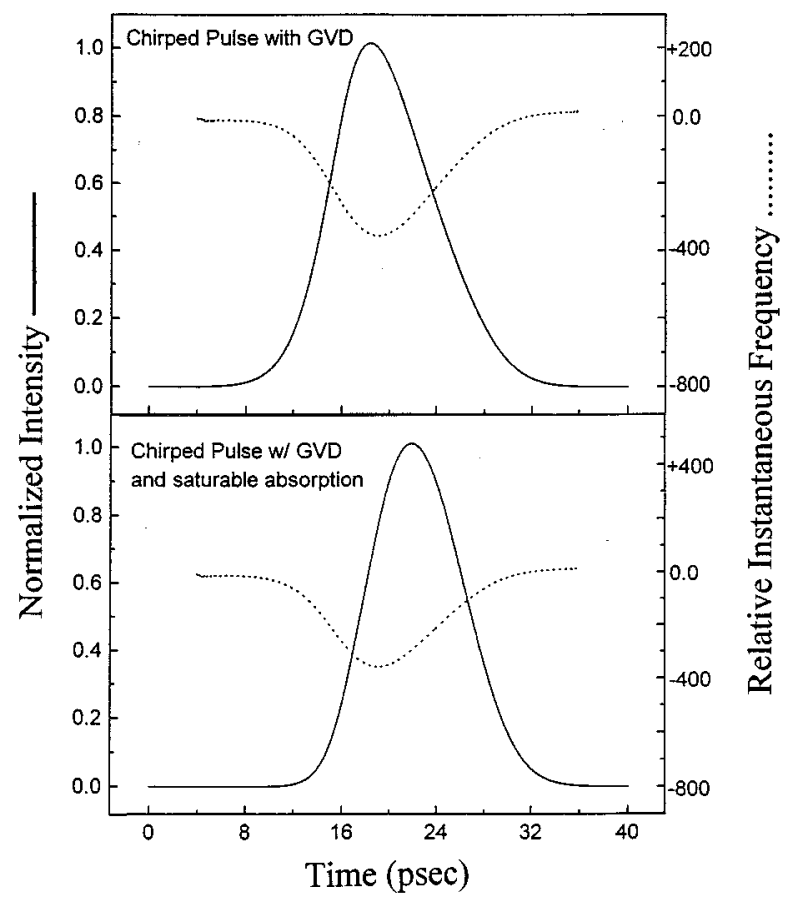

FIG. 5. Intensity pulse profiles and the corresponding instantaneous frequency of pulses: (Top) after a dispersive media with quadratic dispersion, (Bottom) after the intracavity saturable absorber.

optical pulse impinges upon the saturable absorber, the front of the optical pulse is removed. In addition to the resulting decrease of the rise time of the optical pulse, the chirp on the rising edge is also removed, resulting in an optical pulse which not only possesses a fast rising edge, a slow trailing, but also a chirp which is predominantly linear over the main portion of the optical pulse.

These effects can be simulated by considering the optical intensity profiles and their instantaneous frequencies using the nonlinear dynamics described above. It should be noted that the simulation was performed by employing a simple model using normalized nonlinear parameters in order to demonstrate the salient features of the pulse shaping process. In Fig. 5 (top), is the optical pulse shape and its correspond- ing instantaneous frequency after a symmetric pulse has encountered a finite amount of dispersion. Note the asymmetric temporal broadening and the modification of the instantaneous frequency. In Fig. 5 (bottom) are the results after the saturable absorber. Note the faster rising edge of the pulse, as compared to Fig. 5 (top), and also note that the chirp is now predominantly linear over the main portion of the optical pulse. These results suggest that by increasing the intracavity gain, power, and GVD, larger chirping can be obtained, yielding shorter pulses after dispersion compensation. This simple model of SPM, GVD, and saturable absorption supports the experimental results, and provide a clearer understanding of the pulse shaping and chirping dynamics of hybrid mode-locked diode lasers.

In summary, experiments have been performed to measure the intracavity dynamics in order to provide a better understanding of the pulse shaping and chirping dynamics. The important observation is that the integrating nonlinearity leads to asymmetric pulse broadening, while the linearization of the chirp is obtained by the combined effects of GVD and saturable absorption. These results provide a better picture of the pulse production process and chirping mechanisms in mode-locked diode lasers and may enhance the production of ultrashort optical pulses.

This work was supported by the National Science Foundation Grant No. ECS-9410771

${ }^{1}$ K. Hall, J. Mark, E. Ippen, and G. Eisenstein, Appl. Phys. Lett. 56, 1740 (1990).

${ }^{2}$ Y. Lai, K. L. Hall, and E. P. Ippen, IEEE Photonics Technol. Lett. 2, 711 (1990).

${ }^{3}$ P. J. Delfyett, L. T. Florez, N. Stoffel, T. Gmitter, N. C. Andreadakis, Y. Silberberg, J. P. Heritage, and G. A. Alphonse, IEEE J. Quantum Electron. 28, 2203 (1992)

${ }^{4}$ P. W. Smith, Y. Silberberg, and D. A. B. Miller, J. Opt. Soc. Am. B 2, 1228 (1985)

${ }^{5}$ Y. Silberberg, P. W. Smith, D. A. B. Miller, B. Tell, A. C. Gossard, and W. Wiegmann, Appl. Phys. Lett. 16, 701 (1985).

${ }^{6}$ D. J. Kane and R. Trebino, IEEE J. Quantum Electron. 29, 571 (1993).

${ }^{7}$ J. Paye, IEEE J. Quantum Electron. 30, 2693 (1994).

${ }^{8}$ P. J. Delfyett, A. Dienes, J. P. Heritage, M. Y. Hong, and Y. H. Chang, Appl. Phys. B: Lasers Opt. 58, 183 (1994).

${ }^{9}$ P. J. Delfyett, Y. Silberberg, and G. Alphonse, Appl. Phys. Lett. 52, 10 (1991). 